\title{
Tempo de passagem de duas dietas no trato gastrointestinal do peixe-boi da Amazônia Trichechus inunguis (Natterer, 1883) em cativeiro
}

\author{
Paula de Sousa BARBOSA ${ }^{1}$, Vera Maria Ferreira DA SILVA², Geraldo PEREIRA JUNIOR ${ }^{3}$
}

\begin{abstract}
RESUMO
Com o objetivo de testar o tempo de passagem do alimento no trato digestório de peixes-bois da Amazônia em cativeiro, foram testadas separadamente duas dietas distintas. Uma composta exclusivamente de capim do gênero Brachiaria (dieta experimental - DE 1) e outra de capim do gênero Brachiaria acrescentado de pequenas porçôes de ração extrusada para eqüinos (dieta experimental - DE 2). Foram selecionados do plantel do INPA dois animais adultos machos sadios, os quais foram isolados dos demais e submetidos a um período de aclimataçáo às dietas experimentais por 15 dias. Após este período, as dietas foram marcadas com uma fita plástica de $10 \mathrm{~cm}$ e fornecidas aos animais que foram monitorados em intervalos de uma hora. Todo material fecal foi coletado até a recuperação dos marcadores plásticos. A média do tempo de passagem da DE 1 foi de $123 \mathrm{~h} 57 \mathrm{~min}$, cerca de 5,15 dias e da DE 2 foi de $125 \mathrm{~h} 04 \mathrm{~min}$ ou 5,21 dias. Não houve diferença estatística $(\mathrm{P}<0,05)$ entre as dietas fornecidas. $\mathrm{O}$ tempo de passagem observado (aproximadamente 5 dias) coincide com o relatado por outros autores para a espécie, sendo esse tempo considerado uma estratégia para aumentar o tempo de absorçáo nutricional dos alimentos. Apesar do número reduzido de amostras, os resultados sugerem que o uso da ração na alimentação não interfere no tempo de passagem do capim pelo trato digestório do peixe-boi. Com isso, sugere-se que a introdução de alimento concentrado (ração) não afeta a eficiência na digestáo e absorção correta do alimento.
\end{abstract}

PALAVRAS-CHAVE: nutrição, monogástrico, mamífero aquático

\section{Transit time of two diets in the gastrointestinal tract of the Amazonian manatee Trichechus inunguis (Natterer, 1883) in captivity}

\begin{abstract}
The objective of this study was to determine the transit time of two diets in the digestive tract of the Amazonian manatee in captivity. We tested separately two different diets: one composed exclusively of grass of the genus Brachiaria (experimental diet - ED- A 1) and the other composed of grass Brachiaria added with small portions of extruded pellets for horses (experimental diet ED- A 2). Two healthy adult manatees were selected and isolated from the rest and underwent a period of food acclimation with the experimental diets for 15 days. After this period, the experimental diet was marked with a plastic colored tape of 10-cm length and given to the animals. The manatees were monitored at intervals of 1 hour and all fecal material was collected until recovery of the markers. The mean transit time of $\mathrm{ED}$ - A 1 was $123 \mathrm{~h} 57 \mathrm{~min}$, about 5.16 days and ED - A 2 was $125 \mathrm{~h} 04 \mathrm{~min}$ or 5.21 days. There was no statistical difference $(\mathrm{P}<0.05)$ between the transit time of the two diets provided. The transit time observed (approximately 5 days) has also been reported by other authors for this species and is considered a strategy to increase the absorption time of food nutrients. Despite the small sample size, the results suggest that the use of pellets in the diet of the Amazonian manatee did not affect the transit time of the grass. Therefore, it is reasonable to believe that the introduction of concentrated food (pellets) does not affect the efficiency of the animal to digest and absorb food properly.
\end{abstract}

KEYWORDS: nutrition, monogastric, aquatic mammal

${ }^{1}$ Instituto Nacional de Pesquisas da Amazônia-Laboratório de Mamíferos Aquáticos. Av. André Araújo, 2936, Petrópolis, Aleixo, 69083-000 - Manaus, AM - Brasil. Email: manatee_psbd@hotmail.com

2 Instituto Nacional de Pesquisas da Amazônia - Laboratório de Mamíferos Aquáticos. Av. André Araújo, 2936, Petrópolis, Aleixo, $69083-000$ - Manaus, AM - Brasil Email: vmfdasilva@gmail.com

3 Universidade Federal do Amazonas - Coordenação de Biotecnologia. Av. General Rodrigo Octávio Jordão Ramos, 3000, Campus Universitário, Coroado I - Manaus, AM- Brasil Email: geraldoinpa@hotmail.com 


\section{INTRODUÇÃO}

O peixe-boi da Amazônia Trichechus inunguis é um animal exclusivamente herbívoro, que na natureza se alimenta de uma grande variedade de macrófitas aquáticas e semiaquáticas (Best 1981), como por exemplo, Paspalum repens e Echinochloa polystachya, além de gramíneas (Colares e Colares 2002). De acordo com Guterres et al. (2008) provavelmente o peixe-boi também faz uso de folhas, frutos e de matéria orgânica em decomposição no fundo dos lagos durante o período da seca. Ocasionalmente, de forma acidental, o peixe-boi pode vir a ingerir outros elementos como areia e pequenos invertebrados como crustáceos (Colares 1991).

O peixe-boi da Amazônia ingere diariamente cerca de $14 \%$ do seu peso vivo quando jovem e $9 \%$ quando adulto (Best 1981) sendo este consumo altamente influenciado pelo valor nutritivo da dieta, onde fatores como o teor de proteínas, lipídios, glicídios, fibras e umidade parecem atuar como moderadores do consumo alimentar (Cavallante 1995). Outros fatores como a taxa metabólica, fisiologia digestiva da espécie e a palatabilidade do alimento, colaboram na quantidade de alimento ingerido.

T. inunguis é monogástrico capaz de promover a fermentação pós-gástrica dos alimentos, pois é ceco funcional (Burn 1985). Nesta porção do intestino ocorre uma populaçấo microbiana que ataca as fibras e as reduzem em ácidos graxos voláteis (AGV) produzindo energia imediata ou para a síntese de glucose ou gordura (Arruda et al. 2003). Esta particularidade é responsável pela elevada eficiência digestiva da espécie (cerca de 71,1\%) (Burn 1985).

Outro fator importante para essa eficiência digestiva é o tempo de passagem do alimento no trato gastrointestinal, que no caso do T. inunguis é de aproximadamente 120 a 216 horas (5 a 9 dias) (Gallivan e Best 1986). Este intervalo de tempo é reforçado por Itavo (1995) que encontrou um tempo de passagem de 114 a 161 horas, dependendo do tipo de alimento ingerido. Esse tempo de passagem se assemelha ao tempo de passagem encontrado em dugongos (Dugong dugon), que varia de 146 a 166 horas (Lanyon e Marsh 1995) e no peixe-boi da Flórida (Trichechus manatus latirostris) com cerca de 6 a 10 dias (Larkin et al. 2007). O tempo de passagem lento faz com que T. manatus seja um dos herbívoros com maior eficiência de digestão de celulose dentre os mamíferos herbívoros (Burn 1985).

Fisiologicamente, a eficiência da digestão e absorçáo depende do tempo na qual o fluxo do alimento se movimenta ao longo do trato digestório (Clemens e Stevens 1980). Assim, tal estratégia utilizada pelo peixe-boi explica-se por uma taxa de consumo baixa associada a uma velocidade de passagem lenta, aliada a uma digestão pós-gástrica de forrageira bastante eficiente (Lomolino e Ewel 1984).
Estudos realizados com a suplementaçáo de pequenas porçóes de alimentos concentrados da dieta do peixe-boi da Amazônia no Laboratório de Mamíferos Aquáticos - INPA mostraram que uma vez adaptados ao alimento, estes são capazes de consumir satisfatoriamente pequenas porçóes diárias de ração peletizada (P. S. Barbosa_observação pessoal).

$\mathrm{O}$ acréscimo da ração peletizada na dieta do peixe-boi da Amazônia sugere uma nova alternativa alimentar na manutenção desses animais em cativeiro. Uma vez que, nos centros de conservaçáo e pesquisa no Amazonas esses animais são alimentados com capim do gênero Brachiaria além de uma grande variedade de frutos, legumes e verduras, comprados a preço de mercado, o uso da ração poderá diminuir significativamente os custos para manutençáo do peixe-boi em cativeiro.

Rações animais devidamente peletizadas e balanceadas são misturas homogêneas, compostas de diferentes ingredientes analisados quimicamente e balanceados de forma a garantir uma dieta nutricionalmente completa. Além disso, as raçôes permitem um melhor aproveitamento, inclusive de fibras, resultando em muito menos perdas, e consequentemente, uma melhor conversão alimentar (César 1992). Outra vantagem de usar ração na alimentação animal é que o fornecimento de alimentos em pellets também torna mais fácil o manejo e o estoque, diminuindo os custos com transporte, instalações e mão de obra (Rostagno et al. 2007).

A escassez de informaçóes a respeito da administraçáo de alimentos concentrados na dieta de peixes-bois mantidos em cativeiro dificulta a inclusão deste alimento na dieta. Uma informaçâo importante sobre a utilização de concentrado na dieta é o tempo de permanência deste alimento no canal gastrointestinal, já que isso influencia diretamente a maior eficiência alimentar (Silva et al. 2003).

Diante do exposto, objetivou-se com este estudo determinar o tempo de passagem de dois tipos de dieta no trato digestório em peixes-boi da Amazônia em cativeiro.

\section{MATERIAL E MÉTODOS}

O experimento foi conduzido no parque aquático Robin Best, no Instituto Nacional de Pesquisas da Amazônia - INPA, no período de agosto a setembro de 2008, totalizando 60 dias. Foram testadas duas dietas experimentais: dieta volumosa (dieta experimental 1-DE 1) e dieta volumosa e concentrada (dieta experimental 2-DE 2).

Para realização da pesquisa, foram selecionados dois animais machos adultos, em boas condiçóes de saúde (Tapajós - 7 anos e Erê - 10 anos), pesando 99,5 e 118,5 kg; com comprimento total retilíneo (TLSL) de $190 \mathrm{~cm}$ e 180 $\mathrm{cm}$, respectivamente. Para a pesagem dos peixes-bois, foram colocados em uma maca, suspensos por um guincho e cabos 
de aço, utilizando-se uma balança com capacidade de 200 kg subtraindo-se posteriormente o peso da maca. Para medir o comprimento total retilíneo (TLSL) foi utilizado tubo de PVC e corda para ajustar no tamanho do animal e depois essa corda foi medida com fita métrica. Os animais foram isolados dos demais, em um tanque de cambeamento, com medidas aproximadas de 2 metros de profundidade, 2,5 metros de comprimento e 1,5 metros de largura.

Para avaliação da DE 1, houve um período de aclimatação de 15 dias, onde os animais alimentaram-se exclusivamente de capim-colônia (Brachiaria mutica), em uma proporção de $9 \%$ do peso vivo (Best 1981) acrescentado com 10\% a mais sobre o total deste valor a fim de que o consumo fosse ad libitum. Esse processo foi necessário para que o trato gastrointestinal contivesse somente o material da dieta em questão. $\mathrm{O}$ alimento foi fornecido uma vez ao dia, no horário de 17:00 h, respeitando a rotina de alimentaçáo dos animais.

No décimo quinto dia de aclimatação, os animais foram colocados em tanques separados para receberem a dieta experimental 1, que consistiu de porçốes de capim com o marcador amarrado em um maço de folhas. Foi usado como marcador fitas plásticas de cor forte e flexível de aproximadamente $10 \mathrm{~cm}$ de comprimento. Foram quatro marcadores de cores diferentes, sendo duas cores para cada animal. $\mathrm{O}$ alimento foi colocado no tanque e monitorado até que os animais ingerissem a folha marcada. A contagem do tempo foi iniciada a partir da ingestão do marcador $(t=0 \mathrm{~h}) \mathrm{e}$ os animais monitorados de hora em hora a partir do terceiro dia de ingestão. A cada hora todas as fezes na superfície do tanque foram coletadas com um puçá de náilon fino. As fezes eram então esmiuçadas para verificar a presença ou ausência da fita marcadora.

Ao final da avaliação da dieta experimental 1 , os animais foram colocados juntos novamente no tanque de cambeamento para aclimataçáo com a dieta experimental 2. Esta aclimatação teve duração de 15 dias, onde os animais receberam dieta a base de capim-colônia (nas mesmas quantidades da dieta experimental 1), acrescida de ração comercial peletizada para eqüinos. Essa ração era composta basicamente pelos seguintes ingredientes: calcário, farelo de arroz, aditivo antifúngico fungistático, aditivo antioxidante, farelo de trigo, farelo de glúten de milho-60, milho integral moído, melaço, farelo de soja, premix vitamínico mineral, cloreto de sódio (sal comum), lisina, metionina, óleo de soja refinado. A composição nutricional da ração é apresentada na Tabela 2. O alimento foi fornecido uma vez ao dia, no horário de 17:00 h.

Para melhorar a estabilidade física da ração e facilitar a ingestáo pelo peixe-boi, a ração foi submetida a um processo de extrusão. Para isso, a ração peletizada foi inicialmente triturada em um moinho martelo, com malha fina de $0,8 \mathrm{~mm}$, sendo posteriormente misturada com $10 \mathrm{~kg}$ de farinha de trigo, 1 $\mathrm{kg}$ de dextrina e $1,8 \mathrm{~kg}$ de farelo de glúten de milho, para cada $40 \mathrm{~kg}$ de ração.

Em seguida a mistura foi colocada na máquina extrusora a uma pressáo de 30 ATMs e uma temperatura aproximada de $135^{\circ} \mathrm{C}$, para que o amido presente gelatinizasse. A extrusão da mistura ocorreu de forma gradativa atingindo um diâmetro máximo de aproximadamente $2,5 \mathrm{~cm}$. O tamanho de corte da raçâao foi de quatro centímetros de comprimento. A ração extrusada foi seca por dois dias em estufa a uma temperatura de $55^{\circ} \mathrm{C}$, embalada em sacos plásticos e acondicionada em temperatura de $25^{\circ} \mathrm{C}$ até a utilização.

A ração foi introduzida na dieta respeitando as 2.900 calorias diárias recomendas para a espécie (Best 1981). O fornecimento gradativo da ração é apresentado a seguir:

$1^{\circ} \mathrm{a} 5^{\circ}$ dia -50 gramas de raçáo por animal/dia + capimcolônia;

$6^{\circ}$ e $10^{\circ}$ dia -100 gramas de ração por animal/dia + capim-colônia;

$11^{\circ}$ a $15^{\circ}$ - 200 gramas de ração por animal/dia + capimcolônia.

No $15^{\circ}$ dia os animais foram novamente separados para que se pudesse ter controle da ingestão dos marcadores. Foram fornecidos 200 gramas de ração, sendo que quatro "pellets" foram amarrados com fita plástica. A contagem do tempo teve início a partir da ingestão do marcador $(t=0 \mathrm{~h}) \mathrm{e}$ então os animais foram monitorados de hora em hora a partir do terceiro dia de ingestáo. As fezes foram esmiuçadas para verificar a presença ou ausência da fita marcadora.

Para verificar a variação de temperatura ocorrida no tanque ao longo do dia a temperatura da água foi aferida com o uso de um termômetro de mercúrio a uma profundidade de 30 $\mathrm{cm}$. Para diminuir a incidência de radiação solar no tanque foi adaptada uma tenda de lona cobrindo parte do tanque durante o experimento.

Os resultados das repetiçóes feitas com os marcadores plásticos foram analisados no programa Statistica 7.0. Após testar a homogeneidade das variâncias pelo teste de Levene $(\mathrm{P}<0,05)$ os tempos de passagem obtidos entre as duas dietas foram submetidos ao teste $t$ de Student para grupos independentes $(\mathrm{P}<0,05)$.

\section{RESULTADOS}

A média do tempo de passagem da dieta experimental 1 foi de $123 \mathrm{~h} 57 \mathrm{~min}$, equivalente a 5,15 dias, sendo que o primeiro marcador a ser ingerido demorou cerca de $118 \mathrm{~h} 20 \mathrm{~min}$ para ser recuperado nas fezes e o último marcador somente foi recuperado 10 horas depois do primeiro. 
O tempo de passagem da DE-2 (volumoso + concentrado) não variou muito quando comparado com a $\mathrm{DE}-1$ (volumoso), com o tempo médio de passagem de $125 \mathrm{~h} 04 \mathrm{~min}$, equivalente a 5,21 dias, sendo que, não houve diferença estatisticamente significativa no tempo de passagem entre as duas dietas $(\mathrm{P}<$ $0,05)$. Contudo a variaçáo individual foi de aproximadamente 12 horas entre um animal e o outro.

As fezes não apresentaram alteraçôes, no que diz respeito cor e consistência, quando comparadas com os dejetos de outros animais do parque aquático Robin Best, tanto na dieta experimental 1, quanto na dieta experimental 2. Os marcadores foram visualizados nas fezes com relativa facilidade, considerando que todos foram recuperados junto ao bolo fecal no momento da defecação.

A temperatura média da água no tanque no decorrer do experimento foi de $28 \pm 1,5^{\circ} \mathrm{C}$ tanto durante o experimento 1 quanto no experimento 2 . Houveram algumas quedas durante a noite chegando até $26,5^{\circ} \mathrm{C}$ no dia mais frio.

\section{DISCUSSÃO}

O resultado encontrado neste estudo para o tempo de passagem do capim colônia (Brachiaria mutica) foi discretamente superior ao observado por Itavo (1995) que registrou um tempo de passagem de 114 horas, 4,75 dias. Porém, o resultado observado nesta pesquisa corrobora o encontrado por Gallivan e Best (1986) que observaram um tempo médio de 5 dias.

Gallivan e Best (1986) relatam ainda que, este tempo de passagem pode chegar até 9 dias, pois segundo estes autores, o longo período de passagem do bolo alimentar pelo trato gastrointestinal ocorre provavelmente como uma estratégia com o intuito de aumentar a eficiência de digestáo e absorção do alimento. Sendo assim, essa estratégia seria útil principalmente na época de seca dos rios da Amazônia, período em que os animais sofrem com privação de alimentos.

A eficiência digestória em herbívoros não ruminantes aumenta à medida que o tempo de passagem do alimento pelo trato digestório é maior. Isso ocorre, pois a microbiota teria mais tempo para agir e quebrar a celulose, permitindo assim melhor absorção do alimento (Lomolino 1977). De acordo com os resultados aqui apresentados a adiçấo da raçáo na dieta não parece ter alterado o tempo da digestão e consequente absorçấo dos nutrientes contidos no capim.

A velocidade de trânsito do alimento ao longo do canal gastrointestinal relaciona-se, nas diversas espécies animais, ao comprimento do tubo digestório. Assim, velocidade de trânsito aumenta proporcionalmente ao comprimento do intestino (Barbieri et al. 1998). Partindo deste principio, um animal com escore corporal maior sugere-se que possua um trato digestório condizente com seu tamanho. Este fato foi visualizado no presente estudo, quando avaliado a diferença no tempo de passagem individualmente, pois o animal com maior escore corporal (Erê__190 cm) apresentou um tempo de passagem maior quando comparado com o outro indivíduo do experimento de menor porte (Tapajós_180 cm) (Tabela 1). Comparando a taxa de trânsito do alimento no trato digestório de T. inunguis (peixe-boi da Amazônia), de T. manatus latirostris (peixe-boi da Florida) e de Dugong dugon (dugongo), o peixe-boi da Amazônia possui o menor tempo dentre as 3 espécies, provavelmente por ser o menor membro da ordem Sirenia.

A composição nutricional de uma dieta é fator fundamental na regulação do tempo de passagem e regula diretamente a eficiência digestiva do animal (Itavo 1995). Com base neste fato, este trabalho sugere que o peixe-boi da Amazônia aproveita eficientemente a fibra bruta da dieta independente da inclusáo de alimento concentrado. No entanto, deve-se considerar que o tempo de passagem de um alimento não é somente influenciado pela composição deste, mas também pela fisiologia do aparelho digestório e pelo comportamento do animal (Oliveira et al. 2007).

Neste sentido, vale ressaltar que o peixe-boi da Amazônia, a exemplo de outros animais ceco funcional (equinos e elefantes), possui fisiologia digestória para aproveitar de maneira eficiente alimentos ricos em fibra (Burn 1985). Ainda assim, o tempo de passagem de equinos ( 48 a 52 horas) (Oliveira et al. 2003) e de elefantes (21 a 46 horas) (Rees 1982) é bem inferior ao tempo de passagem do alimento no trato digestório do peixe-boi da Amazônia (cerca de 5 dias). Isso provavelmente se deve à menor taxa metabólica de $T$. inunguis (Gallivan e Best 1980).

Para um elemento ser considerado um marcador ele deve ser indigerível e inassimilável pelo organismo, além de não

Tabela 1 - Comparação entre o comprimento total retilíneo (CTR) e o tempo médio de passagem da dieta 1 (TMP1) e dieta 2 (TMP2) em cada animal

\begin{tabular}{lccc}
\hline Animal & CTR $(\mathrm{cm})$ & TMP1 (horas) & TMP2 (horas) \\
\hline Erê & 190 & 124,29 & 130,75 \\
Tapajós & 180 & 122,85 & 119,33 \\
\hline
\end{tabular}

Tabela 2 - Valores nutricionais da ração comercial para equinos, utilizada no experimento.

\begin{tabular}{lc}
\hline Nutrientes & Valor nutricional \\
\hline Proteína bruta (\%) & 15,00 \\
\hline Extrato etéreo (\%) & 3,50 \\
\hline Fibra bruta (\%) & 10,00 \\
\hline Matéria mineral (\%) & 13,00 \\
\hline Cálcio (\%) & 1,50 \\
\hline Fósforo (\%) & 0,50 \\
\hline Umidade (\%) & 13,00 \\
Energia digestível $\left(\mathrm{kcal} \mathrm{kg}^{-1}\right)$ & 3.000 \\
\hline
\end{tabular}


possuir ação farmacodinâmica sobre o trato digestório e não poder se misturar com a digesta (Kavanagh et al. 2001), sendo estas características observadas com os marcadores utilizados neste experimento.

Machado et al. (2008) trabalhando com tempo de passagem em Arctocephalus australis relatam que marcadores sólidos não são eficientes por ficarem retidos por longos períodos no trato digestório dos animais, podendo superestimar os resultados encontrados. Neste contexto sugere-se que estudos utilizando marcadores em pó sejam realizados para que se verifique a ocorrência de variaçôes significativas no tempo de passagem do alimento para o peixe-boi da Amazônia.

A temperatura da água do tanque esteve dentro do recomendado para a espécie (Gallivan et al. 1983). A temperatura observada se deu devido a dois fatores: 1) a renovação constante de água, principalmente nos horários de maior incidência solar no tanque, entre 11 horas até às 15 horas; e 2) à cobertura adaptada para cobrir parte do tanque.

Estudo em bovinos mostrou que animais que se encontram em estresse térmico apresentam déficit de digestibilidade (Fernandes 2005). Em pacus (Piaractus mesopotamicus) esse fator estressante influencia acentuadamente o tempo de trânsito gástrico sendo que quando em temperatura mais baixas o tempo de passagem diminuiu drasticamente (DiasKoberstein et al. 2005).

Por possuir uma taxa metabólica muito baixa o peixe-boi da Amazônia não suporta águas com temperaturas abaixo de $23^{\circ} \mathrm{C}$ (Gallivan et al. 1983). Apesar de o experimento ter sido realizado em região de clima quente e úmido como é o caso do Amazonas, a temperatura da água deve ser monitorada a fim de se evitar que fatores dessa natureza influenciem na homeostase dos animais, comprometendo a taxa de passagem dos alimentos.

\section{CONCLUSÕES}

Os resultados sugerem que o acréscimo de pequenas porçóes de raçáo na dieta do peixe-boi da Amazônia mantido em cativeiro náo altera o tempo de passagem do alimento volumoso pelo trato gastrointestinal, tampouco acarreta transtornos gastrointestinais. Desta forma, acredita-se que a eficiência alimentar dos peixes-bois testados não foi prejudicada pela introdução deste alimento na sua dieta. No entanto, sugere-se que novos estudos sejam realizados sobre a utilização de alimentos concentrados como alternativa alimentar na dieta de peixes-bois da Amazônia em cativeiro.

\section{AGRADECIMENTOS}

Os autores agradecem o apoio logístico do INPA, e o apoio financeiro do programa Petrobras Ambiental/AMPA e CNPq. Agradecemos também ao Dr. Manoel Pereira Filho, a
Dra. Daniela Fettuccia, ao MdV. José Anselmo da A'ffonseca Neto e aos alunos colaboradores: Bruno Bozetti, Louzamira Bivaqua, Laiz Heckman e Gisele Maciel.

\section{BIBLIOGRAFIA CITADA}

Arruda, A. M. V.; Lopes, D. C.; Ferreira, W. M.; Rostagno, H. S.; Queiroz, A. C.; Pereira, E. S.; Silva, J. F.; Jham, G. N. 2003. Atividade microbiana cecal e contribuição da cecotrofia em coelhos alimentados com raçóes contendo diferentes fontes de fibra e níveis de amido. Revista Brasileira de Zootecnia, 32: $891-902$.

Barbieri, R. L.; Leite, R. G.; Stermann, F. A.; Hernandez-Blazquez, F. J. 1998. Food passage time through the alimentary tract of a Brazilian teleost fish, Prochilodus scrofa (Steindachner, 1881) using radiography. Brazilian Journal of Veterinary Research and Animal Science, 35: 32 - 36.

Best, R. 1981. Foods and Feeding Habits of Wild and Captive Sirenia. Mammal Review, 11:3 - 29.

Burn, D. 1985. The Digestive Strategy and Efficiency of the West Indian Manatee Trichechus manatus. Comparative. Biochemistry and Physiology, 85: 139 - 142.

Cavallante, A. 1995. Taxa de Consumo Alimentar do Peixe-boi da Amazônia (Trichechus inunguis) (Natterer, 1883), em Cativeiro. Monografia de conclusão de curso, Universidade de Londrina, Londrina, Paraná. 37 pp.

César, S. 1992. A Importância de uma ração completa e nutricionalmente balanceada para pássaros. Brasil Ornitológico, 2: 59 .

Clemens, E. T.; Stevens, C. E. 1980. A Comparison of gastrointestinal transit time in ten of mammals. Journal Agriculture Science, 94: $735-737$.

Colares, I. 1991. Hábitos Alimentares do Peixe-boi da Amazônia (Trichechus inunguis, Mamalia: Sirenia). Dissertação de Mestrado, Instituto Nacional de Pesquisas da Amazônia/ Fundaçáo Universidade do Amazonas, Manaus, Amazonas. $110 \mathrm{pp}$.

Colares, I.; Colares, E. 2002. Food Plants Eaten by Amazonian Manatees (Trichechus inunguis, Mammalia: Sirenia). Brazilian Archives of Biology and Technology, 45: 67 - 72.

Dias-Koberstein, T. C. R.; Carneiro, D. J.; Urbinati, E. C. 2005. Tempo de trânsito gastrintestinal e esvaziamento gástrico do pacu (Piaractus mesopotamicus) em diferentes temperaturas de cultivo. Acta Scientiarum Animal Sciences, 27: 413 - 417.

Fernandes, A. C. 2005. Efeito do estresse térmico sobre a seleção de dieta por bovinos. Dissertação de mestrado Medicina Veterinária, Universidade do São Paulo, São Paulo- SP. 90 pp.

Gallivan, G.; Best, R. 1980. Metabolism and Respiration of the Amazonian Manatee Trichechus inunguis (Mammalia: Sirenia). Physiological Zoology, 53: 245 - 253.

Gallivan, G; Best, R. 1986. The influence of feeding and fasting on the metabolic rate and ventilation of the Amazonian manatee (Trichechus inunguis). Physiological Zoology, 59: 552 - 557. 
Gallivan, G; Best, R; Kanwisher J. 1983. Temperature regulation in the Amazonian manatee Trichechus inunguis. Physiological Zoology, 56: 255 - 262.

Guterres, M. G.; Marmontel, M.; Ayub, D. M; Singer, R. F. Singer, R. B. 2008. Anatomia e Morfologia de Plantas Aquáticas da Amazônia Utilizadas como Potencial Alimento por Peixe-boi Amazônico. Instituto de Desenvolvimento Sustentável Mamiraua, Amazonas, Brasil. 187 pp.

Itavo, R. 1995. Tempo de Passagem do Alimento no Trato Digestivo do Peixe-boi da Amazônia (Trichechus inunguis) em cativeiro. Monografia de conclusão de curso, Universidade de Londrina, Londrina, Paraná. 35pp.

Kavanagh, S.; Lynch, P. B.; Mara, F. O.; Caffrey, P. J. 2001. A comparison of total collection and marker technique for measurement of apparent digestibility of diets for growing pigs. Animal Feed Science and technology, 89: 49 - 58.

Lomolino, M. 1977. The Ecological Role of the Florida Manatee (Trichechus manatus latirostris) in Water Hyacinth Dominated Ecosystems. Dissertação de mestrado. Universidade da Florida, Gainesville, Florida. 169 pp.

Machado, R.; Wickert, J. C.; Ott, P. H. ; Nakashima, S. B. 2008. Tempo de passagem de alimento através do trato digestório de Arctocephalus australis, estimado a partir de diferentes marcadores In: XII Reunión de Trabajo de Especialistas en Mamíferos Acuáticos de América del Sur $17^{\circ}$ Congresso SOLAMAC, 2008, Montevideo. XII Reunión de Trabajo de Especialistas en Mamíferos Acuáticos de América del Sur / 7o Congresso SOLAMAC, p. $164-164$.

Lanyon J.; Marsh, H. 1995. Digesta Passage Times in Dugong. Australian Journal of Zoology, 43: 119 - 127.
Larkin, I.; Fowler, V.; Reep, R. 2007. Digesta Passage Rates in the Florida Manatee (Trichechus manatus latirostris). Zoo Biology, 26: 503 - 515 .

Lomolino, M; Ewel, K. 1984. Digestive Efficiencies of the West Indian Manatee (Trichechus manatus). Biological Sciences, 3: $176-179$.

Oliveira, C. A. A.; Almeida, F. Q.; Vieira A. A.; Lana, A. M. Q.; Macedo, R.; Lopes, B.A.; Corassa, A. 2003. Cinética de passagem da digesta, balanço hídrico e de hidrogênio em equinos consumindo dietas com diferentes proporçôes de volumoso e concentrado. Revista Brasileira de Zootecnia, 32: 140 - 149.

Oliveira, K.; Costa C.; Faustino, M. G.; Gasque, V. S.; Santos, V. P.; Lima, M. N.; Filho, V. F. N.; Abdalla, A. L. 2007. Valor nutritivo e cinético do trato digestivo de dietas contendo grãos secos ou ensilados de sorgo de baixo e alto tanino para equinos. Revista Brasileira de Zootecnia, 36: 1809 - 1819.

Rees, P. A.; 1982. Gross assimilation efficiency and food passage time in the African elephant. African Journal of Ecology, 20: 193 - 198.

Rostagno, H. S.; Bünzen, S.; Sakomura, N. K.; Albino, L. F. T. 2007. Avanços metodológicos na avaliação de alimentos e exigências nutricionais para aves e suínos. Revista Brasileira de Zootecnia, 36: 295 - 304.

Silva, J. A. M.; Pereira Filho, M.; Oliveira- Pereira, M. I. 2003. Frutos e sementes consumidos pelo tambaqui, Colossoma macropomum (Cuvier, 1818) incorporados em raçóes. Digestibilidade e velocidade de trânsito pelo trato gastrointestinal. Revista Brasileira de Zootecnia, 32: 1815 - 1824.

Recebido em 10/04/2012

Aceito em 18/08/2012 